\title{
Radially propagating fluctuation structures in the scrape-off layer of Alcator C-Mod
}

\author{
O. Grulke \\ MPI for Plasma Physics, EURATOM Association, D-17491 Greifswald, Germany and \\ Ernst-Moritz-Arndt University, D-17489 Greifswald, Germany
}

\author{
J. L. Terry and B. LaBombard \\ Plasma Science and Fusion Center, \\ Massachusetts Institute of Technology, \\ Cambridge, Massachusetts 02139, USA
}

S. J. Zweben

Princeton Plasma Physics Laboratory, P.O. Box 451, Princeton, New Jersey 08540, USA

\begin{abstract}
Radially propagating spatiotemporal fluctuation structures are observed in the scrape-off layer of Alcator C-Mod [I. H. Hutchinson et al., Phys. Plasmas 1(5), 1994] using the combination of electric probes, a radial array of views measuring $\mathrm{D}_{\alpha}$ emission, and two-dimensional imaging of $\mathrm{D}_{\alpha}$ emission. For a specific magnetic field configuration the electric probe and the $D_{\alpha}$ array measured plasma density and potential fluctuations along the same magnetic flux tube. Calculations of the cross-correlation functions of $D_{\alpha}$ intensity fluctuations with ion saturation current fluctuations and floating potential fluctuations, respectively, reveals that the potential associated with fluctuation structures is of dipole-type, consistent with fundamental models for radial blob propagation. Radial and poloidal velocities of fluctuation structures are obtained by two-dimensional spatiotemporal turbulence imaging using an ultra-fast framing camera observing the $\mathrm{D}_{\alpha}$ emission intensity in the poloidal plane. In the poloidal direction the fluctuation structures are predominantly propagating in direction of background $\mathrm{E} \times \mathrm{B}$ velocity. Measured radial velocities are directed outwards with a magnitude of typically $1 \%$ of the ion sound speed.
\end{abstract}




\section{INTRODUCTION}

It is a well known feature that turbulent plasma fluctuations in the scrape-off layer (SOL) of fusion experiments are characterized by sporadic large-amplitude fluctuations in the plasma pressure and the plasma potential. These infrequent fluctuations lead to non-Gaussian probability distribution functions with asymmetric long tails skewed towards larger magnitudes. Recently, the non-Gaussian character of fluctuations has been emphasized by demonstrating that the distribution function of SOL density fluctuations can be described by a gamma distribution [1]. The large-amplitude fluctuations result in a fluctuation induced transport, which shows a strong intermittent character with large transport events contributing significantly to the radial plasma flux [2-4]. Such events are ascribed to spatiotemporal fluctuations structures and the associated transport is caused by radial convection of plasma in potential vortices. [5-7]. In the last years, a different transport mechanism has been observed, in which turbulent fluctuation structures show a direct radial propagation over distances larger than the typical radial structure size [8-11]. These structures are referred to as intermittent transport events(IPOs) [8, 12, 13], avaloids [14], or blobs $[11,15]$ and can directly impact divertor heat loads and wall recycling in tokamak devices. One of the first models to describe the radial propagation properties of blobs was suggested by Krasheninnikov and co-workers. [16, 17]. Here, the radial propagation of blobs is a result of the self-consistent electric field, which is associated with the blob via sheath boundary conditions. Recent advancements of blob modeling also include the transition of blobs across the separatrix [18], magnetic fluctuations associated with blobs [19], and twodimensional global numerical models [20]. Importantly, the different models predict different radial blob velocities, dependent on the spatial scale of blobs, connection lengths to material boundaries, and plasma resistivity. The radial velocity and its scaling is an important piece of information and directly impacts the energy and particle transport associated with blobs. Thus, a detailed characterization of the radial blob dynamics is an important task.

The present paper reports on measurements of the radial propagation velocity of blobs in the SOL of the Alcator C-Mod tokamak [21] using gas-puff turbulence imaging (GPI) diagnostics, which allows for a detailed characterization of the blob evolution and propagation properties. The potential distribution of blobs is determined using the combination of electric probes and a radial array of $\mathrm{D}_{\alpha}$ views. Additionally, distributions of the radial 
TABLE I: Discharge and plasma parameters at three different locations in the SOL: separatrix $(\rho=0 \mathrm{~mm})$, mid-SOL $(\rho=10 \mathrm{~mm})$, and limiter edge $(\rho=20 \mathrm{~mm})$

\begin{tabular}{lccc}
\hline \hline parameter & separatrix mid-SOL limiter edge \\
\hline electron density $n / 10^{19} \mathrm{~m}^{-3}$ & 7 & 3 & 2 \\
electron temperature $T_{e} / \mathrm{eV}$ & 45 & 20 & 10 \\
effective ion gyro radius $\rho_{s} / \mu \mathrm{m}$ & 300 & 200 & 170 \\
ion sound speed $C_{s} / \mathrm{km} \mathrm{s}^{-1}$ & 70 & 50 & 30 \\
\hline \hline
\end{tabular}

and poloidal propagation velocities of blobs are characterized using an ultra-fast camera viewing the $\mathrm{D}_{\alpha}$ emission intensity in a poloidal cross section. The paper is organized as follows: Sec. II outlines the experimental setup and fluctuation diagnostics used to characterize turbulent fluctuations. In Sec. III measurements of the potential distribution of blobs as obtained by correlation measurements parallel to the magnetic field are presented. Sec. IV deals with the radial and poloidal propagation speeds of blobs as obtained by GPI. The results are summarized and discussed in Sec. V.

\section{EXPERIMENTAL SETUP}

The results reported in the present paper were obtained in Ohmic discharges with lower single-null magnetic field configuration. Average plasma densities were typically $\bar{n}=1.5 \cdot 10^{20} \mathrm{~m}^{-3} \approx 0.3 n_{G}$, where $n_{G}$ denotes the Greenwald density limit [22]. The magnetic field was kept constant at a toroidal magnetic field of $B=5.4 \mathrm{~T}$ and a plasma current of $I_{p}=630 \mathrm{kA}$, which results in a safety factor at the $95 \%$ flux surface of $q_{95} \approx 6$. The SOL plasma parameters for the current discharges are compiled in Table I. The choice of the magnetic field configurations is determined in order to measure parallel correlations as it is reported below. To increase statistical confidence, the data are combined from typically six identical discharges. Fluctuations were measured using three complementary diagnostics. The diagnostic arrangement as projected into a poloidal cross section is shown in Fig. 1. A vertically reciprocating multi-tip probe was used to measure simultaneously fluctuations of the ion saturation current and floating potential (1 MHz sampling frequency) as the probe scans across the SOL. If electron temperature fluctuations are assumed to be small, the 


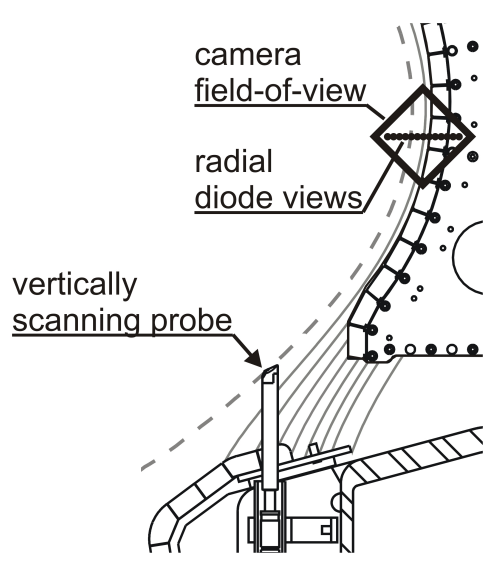

FIG. 1: Cross section showing the camera field-of-view, the radial diode views, and the reciprocating Langmuir probe as projected into the poloidal plane.

signals can be taken as solely dependent on plasma density $\tilde{n}$ and plasma potential fluctuations $\tilde{\Phi}$, respectively. An ultra-fast camera (250 kHz frame-rate, 300 frames memory) views the $\mathrm{D}_{\alpha}$ emission of a localized deuterium gas-puff at the outer mid-plane. The camera's field-of-view (f.o.v.) has a diamond shape and spans over $6 \times 6 \mathrm{~cm}$ radially and vertically, covering the SOL and extending $\approx 3 \mathrm{~cm}$ inside the separatrix. Additionally, a radial array of fiber views ( $\approx 4 \mathrm{~mm}$ object size) is arranged inside the camera's f.o.v., also measuring $\mathrm{D}_{\alpha}$ emission from the gas puff ( $1 \mathrm{MHz}$ sampling frequency). Details of the $\mathrm{D}_{\alpha}$ diagnostic setup can be found in $[10,15,23]$. These may be referred to hereafter as "diode views" or "diode array".

The $\mathrm{D}_{\alpha}$ emission intensity $I$ depends on the neutral gas density $n_{0}$, the electron temperature $T_{e}$, and electron density $n_{e}[24,25]$. However, assuming again small temperature fluctuations and variations of the neutral gas density on a time scale much longer than the turbulent fluctuations, the $\mathrm{D}_{\alpha}$ emission depends only on plasma density fluctuations $\tilde{n}$. If electron temperature fluctuations cannot be neglected they contribute to the amplitudes of ion saturation current and $\mathrm{D}_{\alpha}$ intensity measurements. However, the characterization of the spatiotemporal dynamics of turbulent density fluctuation structures is not affected if the phase between density and electron temperature fluctuations is small. 


\section{PARALLEL STRUCTURE OF FLUCTUATIONS AND POTENTIAL DISTRI- BUTION OF FLUCTUATION STRUCTURES}

It is well known that the turbulent fluctuations are fairly localized perpendicular to the magnetic field (poloidal and radial direction) but extend over much longer distances along the magnetic field, resulting in $k_{\|} / k_{\perp} \ll 1$. This feature is evident when viewing the $\mathrm{D}_{\alpha}$ recycling emission in the poloidal-toroidal plane with the ultra-fast camera. A single camera frame with an exposure time of $6 \mu s$ is shown in Fig. 2. The camera views radially in from outside in this case and is focused such that its f.o.v. is located at the outer mid-plane. A magnetic field line at the radial position of the peak $\mathrm{D}_{\alpha}$ emission intensity, which is located radially at $\rho \approx 1 \mathrm{~cm}$ inside the SOL, is superimposed ( $\rho$ denotes the radial distance to the separatrix as projected to the outer midplane). It is clearly seen that the fluctuations form striations along the magnetic field. The poloidal size of the striations is typically $1 \mathrm{~cm}$, which is much smaller than their extent along the magnetic field line, resulting in long parallel correlation lengths. The fluctuation structures are well aligned with the local magnetic field. Examination of subsequent camera exposures reveals that the striations are not stationary patterns but form, propagate, and finally decay. The striations propagate poloidally mainly in direction of time-averaged background $\mathrm{E} \times \mathrm{B}$ drift (in parallel to the ion diamagnetic drift direction). However, for short times propagation in opposite direction is also observed sometimes, which is likely to be an effect of fluctuations of the radial electric field in the SOL. Based on this field-aligned character of fluctuation structures, the $\mathrm{D}_{\alpha}$ array signals (measuring density fluctuations) are combined with the reciprocating Langmuir probe signals (measuring potential and density fluctuations) to determine the self-consistent potential associated with the fluctuation structures. The $\mathrm{D}_{\alpha}$ diagnostics are located at approximately the same toroidal location, which is on the opposite side of the torus with respect to the probe. For the specified magnetic field configuration and a specific position of the probe in the SOL, the probe maps along a magnetic field line to a certain view of the $\mathrm{D}_{\alpha}$ array. Thus, both diagnostics sample the same field-aligned striation. Fig. 3 shows the magnetic mapping of the probe's plunge to the toroidal location of the radial array of views as reconstructed from EFIT equilibrium calculations. The vertically scanning Langmuir probe intersects with one $\mathrm{D}_{\alpha}$ diode view (here called connecting diode). Note that close to the intersection point a radial change in probe position corresponds also to a change in vertical 


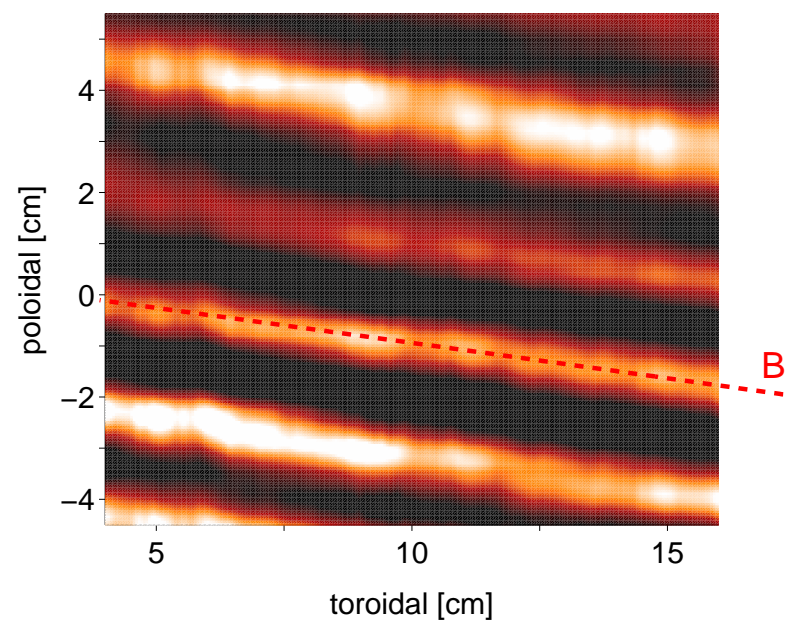

FIG. 2: (Color online) Camera snapshot of $\mathrm{D}_{\alpha}$ intensity striations in a toroidal-poloidal crosssection at the outer mid-plane. A magnetic field line is superimposed at the radial position of maximum $\mathrm{D}_{\alpha}$ emission intensity. The exposure time is $6 \mu s$.

probe position, which is approximately in parallel to the poloidal direction. The connection length along the magnetic field between both diagnostics in the present configuration is $L_{c} \approx$ $3 \mathrm{~m}$. The connection length is typically one order of magnitude larger than the electron-ion collisional mean free path $\lambda_{\mathrm{mfp}} \approx 0.3 \mathrm{~m}$ in the SOL but is much smaller than the distance to material surfaces, which is $15 \mathrm{~m}$ in the mid-SOL for the present configuration. One probe tip is measuring plasma density fluctuations, one tip is measuring plasma potential

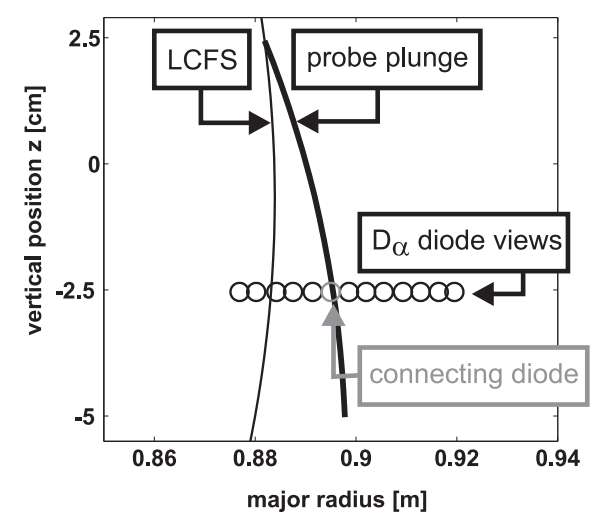

FIG. 3: Poloidal cross section of the SOL showing the position of the $\mathrm{D}_{\alpha}$ diode views, the separatrix, and the mapping of the probe plunge along the magnetic field to the toroidal position of the gas puff. 


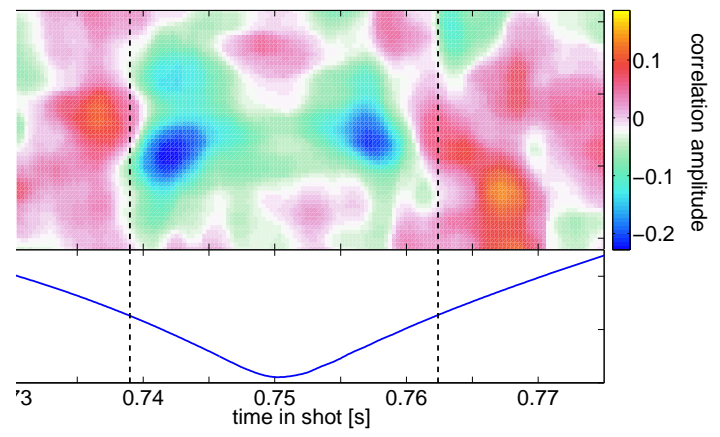

FIG. 4: (Color online) Color-coded plot of the correlation amplitude $C(\tilde{n}, \tilde{\Phi})$ in dependence of the correlation time lag $\tau$ and the time in the shot. Additionally, the position signal of the probe is shown. $\rho$ is the distance to the separatrix

fluctuations. The fluctuation time series are divided into sub-time series, which correspond to a radial motion of the probe by $\Delta \rho=1 \mathrm{~mm}$. For each sub-time series the normalized crosscorrelation function between the $\mathrm{D}_{\alpha}$ intensity fluctuations of the connecting diode (acting as the reference signal $\left.S_{1}=\tilde{n}\right)$ and the two probe tip fluctuation time series $\left(S_{2}=\tilde{n}, \tilde{\Phi}\right)$ are calculated, respectively. The resulting cross-correlation of density and potential fluctuations $C(\tilde{n}, \tilde{\Phi})$ is depicted in Fig. 4. The correlation amplitude is shown, color-coded according

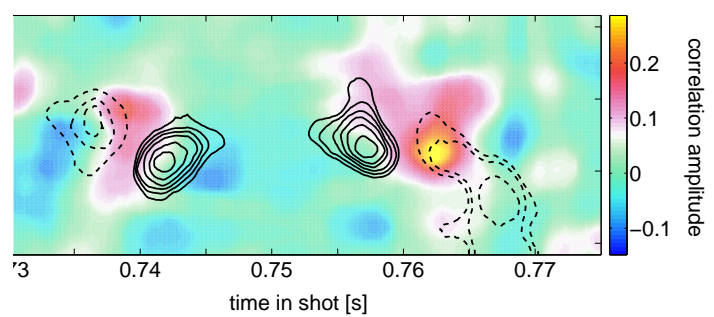

FIG. 5: (Color online) Same as Fig. 4 but showing the correlation amplitudes $C(\tilde{n}, \tilde{n})$. The result from the $C(\tilde{n}, \tilde{\Phi})$ pattern is superimposed as contour plot.

to the correlation time lag $\tau$ for different times in the shot. Additionally, the radial position signal, $\rho$, of the probe inside the SOL is shown (the separatrix is located at $\rho=0$ ). Maximum correlation amplitudes of $\approx 30 \%$ are found. The pattern of the correlation amplitude is of dipole shape with a region of correlation and anti-correlation found during inward and outward probe plunge at the same radial probe position. The dashed lines indicate the approximate radial position of the center of the dipole. The orientation of the dipole is flipped when the probe is moving outwards, indicating a constant spatial orientation of the 
dipole. The correlation during outward motion is less well localized, which is likely a result of perturbations of the potential induced by the conducting probe head. The poloidal extent of the dipole is $\approx 10 \mathrm{~mm}$. Fig. 5 shows the result of correlating the diode signal with the probe measurement of density fluctuations $C(\tilde{n}, \tilde{n})$ in the same representation as Fig. 4 . Similar correlation amplitudes of $30 \%$ are found. However, here the correlation pattern is of monopole-like shape with a single correlation maximum during inward and outward motion of the probe at a specific radial position. Superimposed as a contour plot is the $C(\tilde{n}, \tilde{\Phi})$ dipole correlation pattern from Fig. 4. The monopole correlation pattern lies at the center position of the dipole pattern from Fig. 4. This feature is made more apparent when the respective correlation amplitudes of $C(\tilde{n}, \tilde{n})$ and $C(\tilde{n}, \tilde{\Phi})$, are averaged over the intervals of time lag $\tau$ where the correlation patterns are observed. Fig. 6 shows the average correlation amplitudes over the interval $\tau=[-25 \mu s, 50 \mu s]$ during inward probe plunge. This corresponds to the time interval $t=[0.73 \mathrm{~s}, 0.75 \mathrm{~s}]$ of Figs. 4 and 5 . Based on the mapping calculation (Fig. 3) the radial probe position $\rho$ in Fig. 6 is translated into its vertical position $z$. It is clearly seen that the monopole correlation pattern is located at the center of the dipole pattern. The relative spatial positions of the correlation maxima and minima indicate that the density correlation pattern is phase shifted by $\pi / 2$ relative to the potential pattern. The spatial orientation of the dipole is such that the resulting electric field causes a radially outward oriented $\mathrm{E} \times \mathrm{B}$ drift of the density fluctuation pattern. This finding is consistent with basic models for radial blob propagation, in which the self-consistent potential associated with the plasma pressure perturbation is phase shifted by $\pi / 2$ and also forms a poloidally oriented

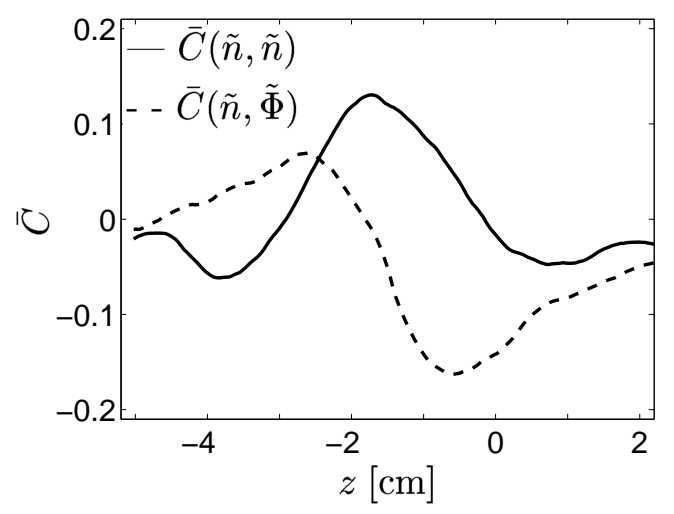

FIG. 6: Cross-correlation amplitudes, time-averaged over the correlation time lag interval $\tau=$ $[-25 \mu s, 50 \mu s]$, vs. the vertical probe position on the SOL $z$. 


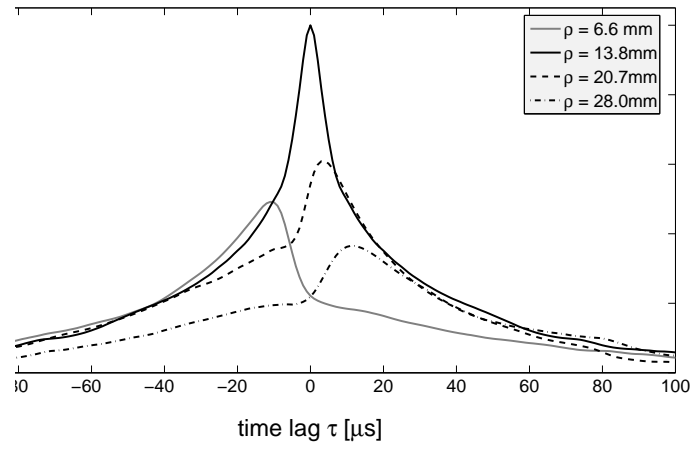

FIG. 7: Cross-correlation functions between different $\mathrm{D}_{\alpha}$ diode time series with the one of a fixed view at $\rho=13.8 \mathrm{~mm}$. For reference the auto-correlation function is also shown as solid black line.

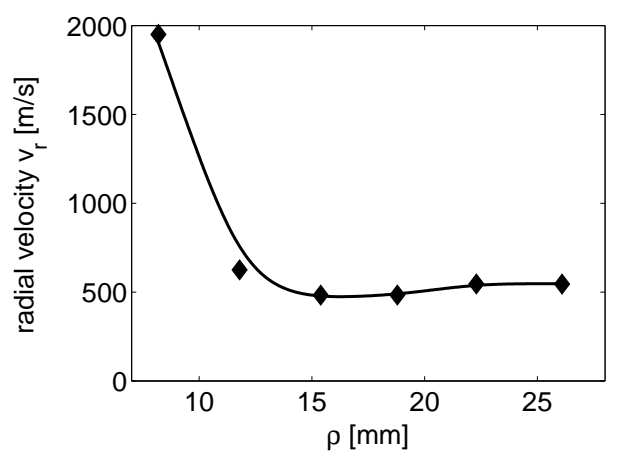

FIG. 8: Radial velocities $v_{r}$ as calculated from the time shift of the peak correlations $\Delta \tau$ and the separation of the diode views $\Delta \rho$.

dipole.

\section{PROPAGATION SPEED OF FLUCTUATION STRUCTURES}

A straightforward approach to measure the radial propagation speed of fluctuations is to inspect the signals from the radial array of $\mathrm{D}_{\alpha}$ diode views. In Fig. 7 the cross-correlation functions of a single diode view located at $13.8 \mathrm{~mm}$ from the separatrix position in the SOL with four other diode views at different locations within the SOL are shown. A relatively large radial correlation length, i.e. the radial distance over which the correlation amplitude decreases to $1 / e$, of $\Delta \rho \approx 15 \mathrm{~mm}$ is found. It is clearly visible from Fig. 7 that the peak values of the respective cross-correlation functions are time shifted. Correlated fluctuations occur consistently earlier at views closer to the separatrix than those located further in the SOL, which corresponds to a radial propagation of fluctuations. From the time shift of 


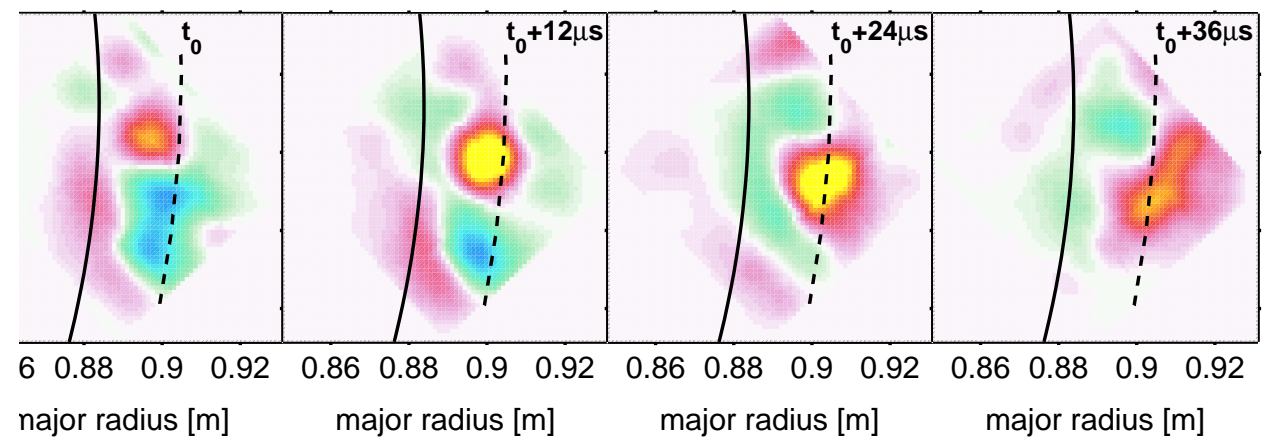

FIG. 9: (Color online) For consecutive ultra-fast camera frames of the $D_{\alpha}$ emission intensity fluctuations in a poloidal cross section. Red and yellow colors correspond to positive fluctuation amplitudes, green and blue to negative. The separatrix position is indicated as the black solid line, the projection of the limiter edge as the dashed line. Shown is the evolution of a long-living blob from its formation close to the separatrix to its decay in the limiter shadow.

the maximum correlation amplitudes, $\Delta \tau$, and the radial separation of the diode views $\Delta \rho$, radial velocities of fluctuations $v_{r}=\Delta \rho / \Delta \tau$ can be deduced. The results for neighboring diode views are shown in Fig. 8. Close to the separatrix, $\rho<10 \mathrm{~mm}$, a high velocity up to $v_{r}=2 \mathrm{~km} / \mathrm{s}$ is found, which may be an artifact of the one-dimensional measurement (s. Sec. V). The velocity drops quickly and remains approximately constant over the rest of the SOL with values $v_{r} \approx 500 \mathrm{~m} / \mathrm{s}$, which corresponds to $v_{r} \approx 1 \% C_{s}$, where $C_{s}$ is the ion sound speed in the SOL. We note that this result is based on a one-dimensional diagnostic, but it is generally observed that the propagation of fluctuations has also a poloidal component [11, 23].

The radial and poloidal component of propagation can be determined simultaneously using the two-dimensional turbulence imaging camera frames. These frames are particularly good at tracking the motion of turbulent structures. In Fig. 9 four consecutive camera frames of $\mathrm{D}_{\alpha}$ intensity fluctuations with a frame-to-frame time separation of $\Delta t=12 \mu \mathrm{s}$ are shown illustrating the formation, propagation, and decay of a long-lived blob. The formation is close to the separatrix in the region of strong plasma pressure gradient. It is clearly visible that the blob has strong poloidal and radial components of propagation as it traverses through the entire SOL into the limiter shadow, where the blob decays. In order to characterize the propagation properties of large amplitude fluctuations, here defined as blobs, the frames are further processed to separate blobs from small amplitude, 

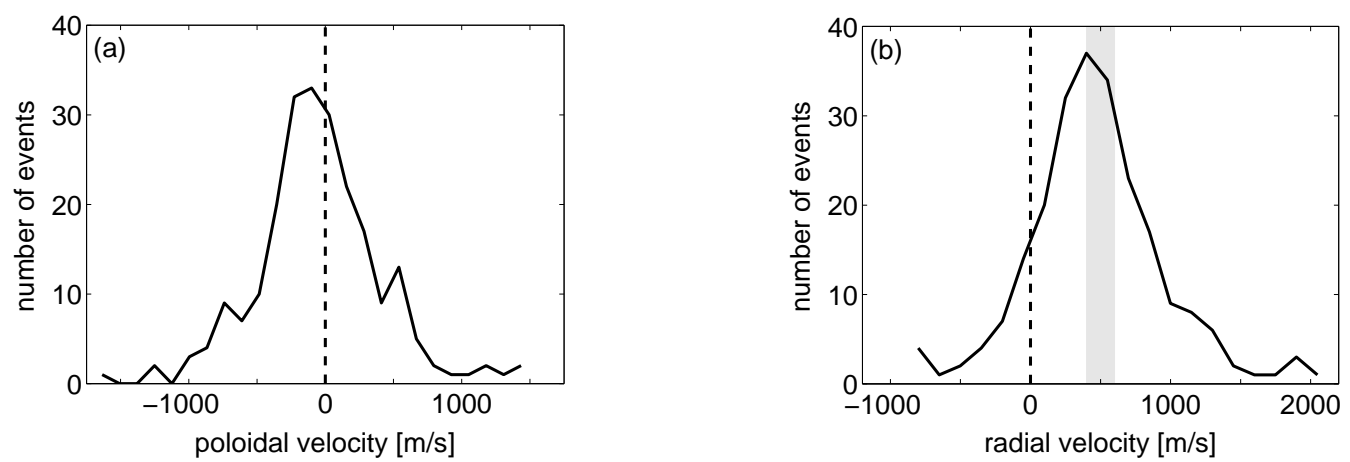

FIG. 10: Number distributions for the poloidal (a) and radial velocity (b) of large-amplitude fluctuation structures as extracted from the camera frames. The shaded area indicates $1 \% C_{s}$ including radial temperature variations in the mid- to far-SOL $(\rho=10 \mathrm{~mm} \ldots 20 \mathrm{~mm})$.

small size fluctuations. The fluctuation time series from each camera pixel is normalized to its respective standard deviation $\sigma$ to account for absolute intensity variations across the f.o.v. of the camera. A threshold to the fluctuation amplitude $I_{\text {thr }}$ and the spatial size $A_{\text {thr }}$ of fluctuations is applied to each individual frame to detect the blobs. The analysis here is done for $I_{\mathrm{thr}}=3 \sigma$ and $A_{\mathrm{thr}}=30$ pixel $=35 \mathrm{~mm}^{2}$ The respective position $(r, z)$ of the blob is defined as the center-of-mass of the blob region. If a different number of blobs are found in two consecutive frames, those blobs are related to each other, whose centerof-mass distance is smallest. The radial $v_{r}$ and poloidal $v_{z}$ velocities are then calculated using the spatial displacement in radial and poloidal direction of the two center-of-mass $(\Delta r, \Delta z)$ and the time between frames $\Delta t$ as $v_{r}=\Delta r / \Delta t$ and $v_{z}=\Delta z / \Delta t$. The number distributions of the two velocity components is shown in Fig. 10. To increase statistical confidence camera movies from six identical discharges are combined, which yields a total number of $\approx 250$ events used for the velocity calculations. The sign convention for the velocities is as follows: negative poloidal velocities are in direction of background poloidal $\mathrm{E} \times \mathrm{B}$ plasma rotation in the SOL as determined by the probe measurements. Positive radial velocities correspond to propagation toward the wall. Both distributions are close to a Gaussian with skewness and kurtosis being $s=0.17, k=1.56$ for $v_{p}$ and $s=0.33, k=1.43$ for $v_{r}$, respectively (note that a Gaussian distribution has $s=k=0$ ). They show a similar broad range of velocities with a full-width-half-maximum of $\approx 1000 \mathrm{~m} / \mathrm{s}$. In the poloidal direction a significant number of events are found propagating in both directions in a range $v_{p} \pm 1000 \mathrm{~m} / \mathrm{s}$. However, the peak is clearly shifted to negative velocities meaning that the 
majority of events are propagating poloidally in background $\mathrm{E} \times \mathrm{B}$ direction. The shift in distribution is much more pronounced for the radial velocities. $90 \%$ of events are found to propagate radially outwards. The maximum velocity found is $v_{r}=2000 \mathrm{~m} / \mathrm{s}$. The peak of the distribution is located at $\approx 500 \mathrm{~m} / \mathrm{s}$, which corresponds to $1 \% C_{s}$ and is similar to the radial propagation speed determined from the radial $\mathrm{D}_{\alpha}$ array. We note that a similar analysis has been also done for negative fluctuations amplitudes and no evidence for inward or outward propagation of negative amplitude fluctuations has been found, independent of the amplitude threshold.

\section{SUMMARY AND DISCUSSION}

The propagation properties of turbulent fluctuations in the SOL of the Alcator C-Mod tokamak using optical $\mathrm{D}_{\alpha}$ diagnostics and electric probes have been studied. Fast camera recordings of $\mathrm{D}_{\alpha}$ intensity fluctuations in both the poloidal-toroidal and the poloidal-radial cross-section visualize the quasi two-dimensional character of fluctuations with an extent perpendicular to the ambient magnetic field that is much smaller than along the magnetic field. Fluctuation structures form striations well aligned with the local magnetic field line. Correlation analysis of the signals from a multi-tip reciprocating Langmuir probe with the signals from a radial array of fast $\mathrm{D}_{\alpha}$ diode views reveals that the potential distribution associated with plasma density fluctuations is of dipole shape. The electric field of the dipole is oriented such that the resulting $\mathrm{E} \times \mathrm{B}$ drift points radially outwards. This finding is consistent with basic models for radial propagation of fluctuation structures and suggests that the turbulence is caused predominantly by curvature driven modes. Significant correlation amplitudes of up to $30 \%$ over a connection length of $3 \mathrm{~m}$ are found for both, density and potential fluctuations, i.e. in $C(\tilde{n}, \tilde{n})$ and $C(\tilde{n}, \tilde{\Phi})$. In comparison to other studies of parallel correlations of fluctuations in tokamak SOL turbulence this maximum correlation amplitude is relatively small $[26,27]$. Several reasons might contribute to a stronger decorrelation of fluctuations in the present situation. The C-Mod SOL plasma is characterized by high collisionality, which leads to a much smaller mean free paths along the magnetic field. Additionally, at the position of the probe the magnetic shear is increased (c.f. Fig. 1), which acts as a decorrelating mechanism for turbulent fluctuations. Finally, the different functional dependence of the probe signal and the $\mathrm{D}_{\alpha}$ intensity fluctuations on plasma density and 
eventual non-zero electron temperature fluctuations might lead to a decreased correlation amplitude.

The cross-correlation functions between the different radial views of the $\mathrm{D}_{\alpha}$ diodes clearly indicate a radial propagation of fluctuations with a typical propagation speed of $\approx 500 \mathrm{~m} / \mathrm{s}$ in the mid- and far-SOL. The diodes closer to the separatrix, the region where stronger poloidal flows than in the mid- and far-SOL are generally observed, provide a higher radial propagation speed. It is well known that in the case of predominantly poloidal propagation of fluctuation structures, measurements with only radial resolution reveal artificially high radial propagation speeds [5]. Thus, the detected high velocity within $\rho \leq 10 \mathrm{~mm}$ is likely to be an artifact of the one-dimensional diagnostic approach. Over a wide range of the SOL the radial velocity stays constant and no indications for radial acceleration or deceleration are found. This finding stands in contrast to radial propagation speeds measured in the SOL of the DIII-D tokamak [8], where the radial velocity of fluctuations was found to decrease by one order of magnitude continuously between the separatrix position and the far-SOL.

The radial velocities deduced from calculation of cross-correlation functions are averaged over the entire fluctuation spectrum. Analysis of the ultra-fast camera frames has characterized the propagation properties of plasma blobs, i.e. fluctuation structures with amplitudes exceeding $3 \sigma$. Distribution functions of both poloidal and radial velocities are obtained simultaneously. The poloidal velocity distribution is clearly shifted in direction of background $\mathrm{E} \times \mathrm{B}$ drift. The peak of the distribution is found at relatively small poloidal velocities, while the shift of the distribution is more pronounced for the radial velocities, indicating that the blob propagation is predominantly radial. The shift of the distribution is more pronounced for the radial velocities. $90 \%$ of events are propagating radially outward with a mean velocity of $v_{r} \approx 1 \% C_{s}$. This corresponds fairly well with the radial velocity obtained from the radial $\mathrm{D}_{\alpha}$ diode correlation analysis, which indicates that blobs dominate the correlation amplitude.

\section{Acknowledgments}

This work is supported by US DoE Coop. Agreement DE-FC02-99ER54512. One author (O. G.) thanks the Alexander-von-Humboldt foundation for the financial support in the 
framework of a Feodor Lynen Fellowship.

[1] J. P. Graves, J. Horacek, R. A. Pitts, and K. I. Hopcraft, Nucl. Fusion 47, L1 (2005).

[2] B. A. Carreras, V. E. Lynch, D. E. Newman, R. Balbin, J. Bleuel, M. A. Pedrosa, M. Endler, B. v. Milligen, E. Sanchez, and C. Hidalgo, Phys. Plasmas 7(8), 3278 (2000).

[3] E. Sánchez, C. Hidalgo, D. López-Bruna, I. Garcia-Cortés, R. Balbin, M. A. Pedrosa, B. v. Milligen, C. Riccardi, G. Chiodini, J. Bleuel, M. Endler, B. A. Carreras, et al., Phys. Plasmas $7(5), 1408$ (2000).

[4] B. A. Carreras, V. E. Lynch, and B. LaBombard, Phys. Plasmas 8(8), 3702 (2001).

[5] J. Bleuel, M. Endler, H. Niedermeyer, M. Schubert, H. Thomsen, and the W7-AS team, New J. Phys 4, 38.1 (2002).

[6] O. Grulke and T. Klinger, New J. Phys 4, 67.1 (2002).

[7] O. Grulke, T. Klinger, and A. Piel, Phys. Plasmas 6(3), 788 (1999).

[8] J. A. Boedo, D. L. Rudakov, R. A. Moyer, G. R. McKee, R. J. Colchin, M. J. Schaffer, P. G. Stangeby, W. P. West, S. L. Allen, T. E. Evans, R. J. Fonck, E. M. Hollmann, et al., Phys. Plasmas 10(5), 1670 (2003).

[9] J. L. Terry, R. Maqueda, C. S. Pitcher, S. J. Zweben, B. LaBombard, E. S. Marmar, A. Y. Pigarov, and G. Wurden, J. Nucl. Mater. 290-293, 757 (2001).

[10] S. J. Zweben, D. P. Stotler, J. L. Terry, B. LaBombard, M. Greenwald, M. Muterspaugh, C. S. Pitcher, the Alcator C-Mod Group, K. Hallatschek, R. J. Maqueda, B. Rogers, J. L. Lowrance, et al., Phys. Plasmas 9(5), 1981 (2002).

[11] S. J. Zweben, R. J. Maqueda, D. P. Stotler, A. Keese, J. Boedo, C. E. Bush, S. M. Kaye, B. LeBlanc, J. L. Lowrance, V. J. Mastrocola, R. Maingi, N. Nishino, et al., Nucl. Fusion 44, $134(2004)$.

[12] J. A. Boedo, D. Rudakov, R. Moyer, S. Krasheninnikov, D. Whyte, G. McKee, G. Tynan, M. Schaffer, P. Stangeby, P. West, S. Allen, T. Evans, et al., Phys. Plasmas 8(11), 4826 (2001).

[13] D. L. Rudakov, J. A. Boedo, R. A. Moyer, S. Krasheninnikov, A. Leonard, M. A. Mahdavi, G. R. McKee, G. D. Porter, P. C. Stangeby, J. G. Watkins, W. P. West, D. G. Whyte, et al., Plasma Phys. Controlled Fusion 44, 717 (2002).

[14] G. Y. Antar, P. Devynck, X. Garbet, and S. C. Luckhardt, Phys. Plasmas 8(5), 1612 (2001). 
[15] J. L. Terry, S. J. Zweben, K. Hallatschek, B. LaBombard, R. J. Maqueda, B. Bai, C. J. Boswell, M. Greenwald, D. Kopon, W. M. Nevins, C. S. Pitcher, B. N. Rogers, et al., Phys. Plasmas 10(5), 1739 (2003).

[16] D. A. D'Ippolito, J. R. Myra, and S. I. Krasheninnikov, Phys. Plasmas 9(1), 222 (2002).

[17] D. A. D'Ippolito, J. R. Myra, S. I. Krasheninnikov, G. Q. Yu, and A. Y. Pigarov, Contrib. Plasma Phys. 44(1-3), 205 (2004).

[18] D. Russell, D. A. D’Ippolito, J. R. Myra, W. M. Nevins, and X. Q. Xu, Phys. Rev. Lett. 93, 265001 (2004).

[19] S. I. Krasheninnikov, A. I. Smolyakov, G. Yu, and T. K. Soboleva, Czech. J. Phys. 55, 307 (2005).

[20] O. E. Garcia, V. Naulin, A. H. Nielsen, and J. J. Rasmussen, Phys. Rev. Lett. 92(16), 165003 (2004).

[21] I. H. Hutchinson, R. Boivin, F. Bombarda, P. Bonoli, S. Fairfax, C. Fiore, J. Goetz, S. Golovato, R. Granetz, M. Greenwald, S. Horne, A. Hubbard, et al., Phys. Plasmas 1(5), 1511 (1994).

[22] M. Greenwald, J. L. Terry, S. M. Wolfe, S. Ejima, M. G. Bell, S. M. Kaye, and G. H. Neilson, Nucl. Fusion 28(12), 2199 (1988).

[23] J. L. Terry, S. J. Zweben, B. Bose, O. Grulke, E. S. Marmar, J. Lowrance, V. Mastrocola, and G. Renda, Rev. Sci. Instrum. 75(10), 4196 (2004).

[24] R. J. Maqueda, G. A. Wurden, D. P. S. ans S. J. Zweben, B. LaBombard, J. L. Terry, J. L. Lowrance, V. J. Mastrocola, G. F. Renda, D. A. D'Ippolito, J. R. Myra, and N. Nishino, Rev. Sci. Instrum. 74(3), 2020 (2003).

[25] R. J. Maqueda, G. A. Wurden, S. Zweben, L. Roquemore, H. Kugel, D. Johnson, S. Kaye, S. Sabbagh, and R. Maingi, Rev. Sci. Instrum. 72(1), 931 (2001).

[26] H. Thomsen, M. Endler, J. Bleuel, A. V. Chankin, S. K. Erents, G. F. Matthews, and Contributors to the EFDA-JET workprogramme, Phys. Plasmas 9(4), 1233 (2002).

[27] C. P. Ritz, E. J. Powers, T. L. Rhodes, R. D. Bengtson, K. W. Gentle, H. Lin, P. E. Phillips, A. J. Wootton, D. L. Brower, N. C. Luhmann, Jr., W. A. Peebles, et al., Rev. Sci. Instrum. 59(8), 1739 (1988). 